\title{
Mitteilungen
}

\section{Schweizerische Neurologische Gesellschaft}

\section{Preis der Mogens und Wilhelm Ellermann-Stiftung}

Die Schweizerische Neurologische Gesellschaft verleiht 2018 zum sechzehnten Mal den Preis der Mogens und Wilhelm Ellermann-Stiftung im Betrage von 20000 CHF.

Der Preis wird vergeben

a) für eine wissenschaftliche Arbeit aus dem Gebiet der neurologischen Wissenschaften, die in den letzten zwei Jahren publiziert oder von einer international anerkannten Zeitschrift zur Publikation angenommen worden ist,

b) für eine Monographie, die nicht den Charakter eines Lehrbuches oder einer Übersicht hat, oder

c) für ein wissenschaftliches Gesamtwerk aus dem Gebiet der neurologischen Wissenschaften.

Die Arbeit muss von einem oder mehreren Schweizer Wissenschaftern oder von Ausländern, die an einer schweizerischen Klinik oder Institut tätig sind, verfasst worden sein. Vorzugsweise sollen jüngere Autoren, die nicht oder erst kürzlich habilitiert worden sind, berücksichtigt werden.

Bewerbungen mit Curriculum vitae und Publikationsliste (nur peer-reviewed Originalarbeiten) in fünffacher Ausführung inkl. Separata sind bis zum $30.11 .2017 \mathrm{zu}$ richten an Prof. Dr. med. C. L. Bassetti, Direktor und Chefarzt, Universitätsklinik für Neurologie, Inselspital Bern, 3010 Bern.
Schweizerische Gesellschaft für Allgemeinchirurgie und Traumatologie / Société Suisse de Chirurgie Générale et de Traumatologie

Vorstand/Comité SGACT/SSCGT 2017

Präsident/Président

Dr. med. Michele Arigoni, Locarno

Past-Präsident/Past Président

Dr. med. Philipp M. Lenzlinger, Schlieren

Sekretär/Sécretaire

PD Dr. med. Urs Neff, Schaffhausen

Quästor/Caissier

Dr. med. Tobias Zingg, Lausanne

Vorstandsmitglieder/Membres du Comité

Dr. med. Thomas Beck, Visp

Dr. med. Karin Bläuer, Biel

Dr. med. Michael Dietrich, Zürich

Dr. med. Andrea Donadini, Lugano-Besso

Dr. med. Jürg Gurzeler, Zofingen

Dr. med. Thomas Hotz, Winterthur

Dr. med. Rebecca Kraus, Aarau

Dr. med. Franziska Saxer, Basel

Dr. med. Christoph Sommer, Chur

SGACT-Sekretariat/Secrétariat SSCGT

c/o Meister ConCept GmbH

Bahnhofstrasse 55

CH-5001 Aarau

$\mathrm{T}+41628362090$

F+41628362097

sgact@meister-concept.ch

www.sgact.ch 